\title{
Als die Volkskammer (fast) zum Parlament wurde ... „Sozialistischer Parlamentarismus“ in der DDR 1989/90*
}

\author{
Uwe Kranenpohl
}

\begin{abstract}
„Wir haben neuen Umgang gepflegt mit unseren Wählern, mit der Regierung der nationalen Verantwortung. Das hat die Arbeit der Ausschüsse, der Fraktionen nun in ihren eigentlichen Sinn erhoben, das hat die Parlamentsdebatte völlig verändert. Ja, ohne Großspurigkeit möchte ich sagen, innerhalb von Tagen wurden wir vom Abstimmungsparlament zu einer arbeitenden Körperschaft, die auch für die eigene und internationale Presse von höchster Aktualität wurde. Die Arbeit unserer Volkskammer rückte in den Mittelpunkt politischer Medienarbeit."1 Dieses Fazit weniger turbulenter Monate wählte Präsident Günther Maleuda am 7. März 1990 anlässlich der letzten Tagung der 9. Volkskammer der DDR. Er bezog sich damit auf die tief greifenden politischen Umwälzungsprozesse, die seit dem Herbst 1989 die ganze Republik erfasst und selbstverständlich auch nicht vor dem zumindest gemäß den Buchstaben des Art. 48 DDR-Verfassung von 1974 (DDR-V) „oberste[n] staatliche[n] Machtorgan der Deutschen Demokratischen Republik“ haltgemacht hatten. Die Volkskammer war 1986 noch über Einheitslisten der Nationalen Front "gewähl" worden und hatte sich selbstverständlich der in Art. 1 DDR-V postulierten Führungsrolle der SED zu unterwerfen. Gelang es ihr trotzdem - wie Maleuda konstatierte -, sich von einer „sozialistischen Vertretungskörperschaft“ zu einem Parlament im liberalen Sinne zu wandeln?

Durchaus überraschend erscheint, dass die hier untersuchte kurze, aber ereignisreiche Phase deutscher Parlamentsgeschichte noch nicht systematisch untersucht worden ist. ${ }^{2} \mathrm{Al}-$ lerdings liegen auch zur „sozialistischen Vertretungskörperschaft“ vor 1989 nur wenige Studien vor $^{3}$, und die freigewählte Volkskammer 1990 wurde bisher ebenfalls nur sehr stief-
\end{abstract}

* Ich danke Harald Braumann, Henrik Gast, Dominik Hammer, Ondřej Kalina und Carola Vogel für vielfältige Verbesserungsvorschläge.

1 Volkskammer der DDR, Stenographischer Bericht (StB), 9. Wahlperiode, S. 555.

2 Anzuführen ist neben einer Analyse von Peter Joachim Lapp, die allerdings ausdrücklich den Stand des Jahreswechsels 1989/90 widerspiegelt, lediglich ein Festschriftenbeitrag von Heinrich Oberreuter sowie einige Passagen in den Stellungnahmen von Zeitzeugen in der Tagungsdokumentation von Werner J. Patzelt und Roland Schirmer. Vgl. Peter Joachim Lapp, Anspruch und Alltag der Volkskammer vor dem Umbruch 1989/90, in: ZParl, 21. Jg. (1990), H. 1, S. 115 125; Heinrich Oberreuter, Vom „sozialistischen“ zum demokratischen Parlamentarismus, in: Jürgen Hartmann / Uwe Thaysen (Hrsg.), Pluralismus und Parlamentarismus in Theorie und Praxis, Opladen 1992, S. 285 - 297; Werner J. Patzelt / Roland Schirmer (Hrsg.), Die Volkskammer der DDR. Sozialistischer Parlamentarismus in Theorie und Praxis, Wiesbaden 2002. Das laufende Projekt „Die Reinstitutionalisierung der DDR-Volkskammer als Parlament im modernen Sinn" der Kommission für Geschichte des Parlamentarismus und der politischen Parteien lässt aber eine deutliche Verbesserung der Forschungslage erwarten (http://www.kgparl.de/projektparl-ddr.html, Abruf am 14. August 2009).

3 Diese erschienen zum größten Teil aber noch vor der Wende: Eckhard Jesse, Die Volkskammer der DDR: Befugnisse und Verfahren nach Verfassung und politischer Praxis, in: Hans-Peter Schneider / Wolfgang Zeh (Hrsg.), Parlamentsrecht und Parlamentspraxis in der Bundesrepublik Deutschland, Berlin / New York 1989, S. 1821 - 1844; Peter Joachim Lapp, Die Volkskammer der DDR, 
mütterlich behandelt. ${ }^{4}$ Die folgende Analyse, mit der diese Forschungslücke teilweise geschlossen werden soll, basiert auf den neun Plenartagungen der Volkskammer zwischen dem 24. Oktober 1989 (10. Tagung) und 6./7. März 1990 (18. Tagung). ${ }^{5}$

\section{Status und Organisation der Volkskammer}

Die Volkskammer war nach der in der DDR herrschenden Ideologie kein „Parlament“, denn Parlamente dienten in deren Verständnis der „Bourgeoisie [dazu], ihre Herrschaft demokratisch zu verbrämen und den Klassencharakter des Staates zu verschleiern “6. Anders als in liberalen Demokratien unterlagen sozialistische Volksvertretungen auch nicht der Gewaltenteilung, also der Kontrolle, Hemmung und Begrenzung durch andere Machtträger, sondern durften „von niemandem in ihren Rechten eingeschränkt werden "7. Demzufolge firmierten alle anderen staatlichen Institutionen der DDR - Ministerrat, Staatsrat, Nationaler Verteidigungsrat, aber selbstverständlich auch alle Organe der Rechtspflege ${ }^{8}-$ als Organe der Volkskammer und waren dieser de jure stets rechenschaftspflichtig. Daraus ergab sich nicht nur eine umfassende formale Machtfülle der Volkskammer, sondern auch eine andere Funktionsbeschreibung als für ein „bürgerliches“ Parlament: „Als arbeitende Körperschaften treffen die sozialistischen Vertretungsorgane nicht nur die maßgeblichen Entscheidungen zur Leitung des Staates, sie haben vielmehr auch zu organisieren und zu sichern, dass ihre Entscheidungen durch alle Staatsorgane und im gesamten Leben der Gesellschaft verwirklicht werden. Die Abgeordneten haben an der Organisation und Kontrolle der Durchführung selbst teilzunehmen."

Opladen 1975; Gero Neugebauer, Die Volkskammer der DDR, in: ZParl, 5. Jg. (1974), H. 3, S. 386 - 411; Heinrich Oberreuter, Sozialistischer Parlamentarismus? Idee, Norm und Realität sozialistischer Vertretungskörperschaften in vergleichender Sicht, in: Jürgen Weber (Hrsg.), Konflikt und Integration III, München 1980, S. 213 - 250; sowie mit Blick in den gesamten Ostblock: Siegfried Lammich, Grundzüge des sozialistischen Parlamentarismus, Baden-Baden 1977. Nach der Wende war insbesondere die Dresdner Arbeitsgruppe um Werner J. Patzelt und Roland Schirmer aktiv. Vgl. dies. (Hrsg.), a.a.O. (Fn. 2); außerdem Heinrich Oberreuter, Parlamentsverständnis, in: Rainer Eppelmann u.a. (Hrsg.), Lexikon des DDR-Sozialismus, Paderborn 1997, S. $608-613$.

4 Vgl. Richard Schröder / Hans Misselwitz (Hrsg.), Mandat für die deutsche Einheit. Die 10. Volkskammer zwischen DDR-Verfassung und Grundgesetz, Opladen 2000. Dazu jüngst auch Roland Schirmer, Die letzte Volkskammer der DDR - ein Parlament ohne Zeit?, in: Werner J. Patzelt I Stephan Dreischer (Hrsg.), Parlamente und ihre Zeit. Zeitstrukturen als Machtpotentiale, BadenBaden 2009, S. 123 - 155.

5 Das Debattenprotokoll dieser Sitzungen umfasst über 300 Druckseiten (StB 9/221-556).

6 Stichwort „Parlamentarismus“, in: Gertrud Schütz u.a., Kleines politisches Wörterbuch, Berlin (Ost) 1989, S. 734; vgl. zur Anfangszeit der DDR aber Joachim Lapp, a.a.O. (Fn. 3), S. 27 - 31.

7 Stichwort „Volkskammer“, in: Gertrud Schütz u.a., a.a.O. (Fn. 6), S. 1048.

8 Vgl. Peter Joachim Lapp, Der Ministerrat der DDR. Aufgaben, Arbeitsweise und Struktur der anderen deutschen Regierung, Opladen 1982; ders., Der Staatsrat im politischen System der DDR (1960-1971), Opladen 1972; Otto Wenzel, Kriegsbereit. Der Nationale Verteidigungsrat der DDR 1960 bis 1989, Köln 1995; Hubert Rottleuthner / Andrea Baer / Wolfgang Behlert I Andrea Feth / Andreas Gängel / Werner Künzel / Thomas Lorenz / Falco Werkentin, Steuerung der Justiz in der DDR, Köln 1994.

9 Herbert Kelle / Tord Riemann, Die Volkskammer - wie sie arbeitet, Berlin (Ost) 1989, S. 16 - 18. 
Zur Wahrnehmung ihrer Aufgaben konnte die Volkskammer aber nicht nur auf die von ihr eingesetzten eigenständigen Organe zurückgreifen, sondern auch auf interne arbeitsteilige Strukturen. So bestanden 1989 zwölf Ausschüsse für unterschiedliche Politikfelder sowie Ausschüsse für Eingaben der Bürger, für Geschäftsordnungsfragen und für Mandatsprüfung. Geleitet wurden die Geschäfte der Volkskammer vom 13-köpfigen Präsidium. ${ }^{10} \mathrm{Zu}$ dem organisierten sich die Abgeordneten entlang der Parteien und Massenorganisationen, durch die sie entsandt worden waren, in Fraktionen.

Allerdings unterlag die Volkskammer als sozialistische Vertretungskörperschaft selbstverständlich den ideologischen Vorgaben der marxistisch-leninistischen SED, deren Führungsrolle in Art. 1 DDR-V unmissverständlich festgeschrieben war. ${ }^{11}$ Zusätzlich abgesichert wurde diese durch den Modus der „Wahl“ der Abgeordneten über die Einheitslisten der Nationalen Front. ${ }^{12}$ So dominierten SED-Mitglieder auch in den Fraktionen der in der Volkskammer vertretenen Massenorganisationen, die neben der SED und den Blockparteien Christlich-Demokratische Union (CDU), Liberaldemokratische Partei Deutschlands (LDPD), Demokratische Bauernpartei Deutschlands (DBD) und Nationaldemokratische Partei Deutschlands (NDPD) immerhin über ein Drittel der Mandate verfügten, denn selbstverständlich waren Funktionsträger des Freien Deutschen Gewerkschaftsbundes (FDGB), der Freien Deutschen Jugend (FDJ), des Demokratischen Frauenbundes Deutschlands (DFD), des Kulturbundes (KB) und der Vereinigung der gegenseitigen Bauernhilfe (VdgB) üblicherweise SED-Mitglieder - wie es auch für Mitglieder der SED-Fraktion selbstverständlich war, einschlägigen Massenorganisationen anzugehören. ${ }^{13}$ Und natürlich galt auch für die Fraktionen der Blockparteien, dass diese nur in äußerst geringem Maße eigene Initiativen entwickeln durften: „Eigenheiten waren möglich, wo es darum ging, die Politik der SED verständlich zu machen, nicht aber, um sich als eigenständige Partei zu profilieren. Konkurrenz zum Parteiwillen der SED durfte es also nicht geben, sehr wohl aber Unterschiedlichkeit in dessen Vermittlung. " 14

Der sozialistische Charakter der Volkskammer zeigte sich auch im Abgeordnetenverständnis. Die Amtspflichten des Abgeordneten sollten nämlich „unter voller Beibehaltung seiner beruflichen Tätigkeit erfüllt [werden]. [...] Man vertritt die Auffassung, dass die Vertretungsorgane nur dann ,arbeitende Körperschaften' sein könnten, wenn sie sich aus Abgeordneten zusammensetzen, die unmittelbar in der Produktion oder in anderen Bereichen des gesellschaftlichen Lebens beschäftigt sind"15. Ausfluss ideologischer Prädispositionen war zudem, dass die Abgeordneten in ihren Entscheidungen nicht frei, sondern mit

10 Vgl. ebenda, S. 57, S. 90.

11 In diesem Kontext sollte zumindest kurz darauf hingewiesen werden, dass nach marxistisch-leninistischer Auffassung der sozialistische Staat dem Schutz gegen Angriffe der Konterrevolution zu dienen hatte, weshalb die umfassenden Kompetenzen sozialistischer Vertretungskörperschaften auch ein gesellschaftlich repressives Moment enthalten.

12 Vgl. Peter Joachim Lapp, Wahlen in der DDR. Wählt die Kandidaten der Nationalen Front!, Berlin 1982.

13 Vgl. dazu die Biographien im Abgeordnetenhandbuch: Die Volkskammer der Deutschen Demokratischen Republik. 9. Wahlperiode, Berlin (Ost) 1987. Vgl. zu den Massenorganisationen allgemein Rüdiger Henkel, Im Dienste der Staatspartei: Über Parteien und Organisationen in der DDR, Baden-Baden 1994.

14 Günter Hartmann, Fraktionen: parlamentarische Rudimente oder Gremien mit Bedeutung, in: Werner J. Patzelt / Roland Schirmer, a.a.O. (Fn. 2), S. 206 - 214, S. 207.

15 Siegfried Lammich, a.a.O. (Fn. 3), S. 87. 
einem imperativen Mandat ausgestattet waren - allerdings tat sich selbst die einschlägige sozialistische Literatur bei der Frage schwer, welche Konsequenzen daraus konkret zu ziehen seien. ${ }^{16}$ Eine Folge war die - allerdings nicht auf sozialistische Volksvertretungen beschränkte ${ }^{17}$ - Möglichkeit der Wähler, gemäß Art. 57 II DDR-V Abgeordnete abzuberufen, die ihre Pflichten „gröblich verletzen“. Dieses Recht der Wähler wurde aber „durch die einfache Gesetzgebung in das Recht des Vertretungsorgans, dem Abgeordneten das Mandat zu entziehen, umgewandelt"18 ( $\$ 47$ IV Wahlgesetz 1976). ${ }^{19}$ Faktisch verkam es damit zu einem Disziplinierungsinstrument der kommunistischen Partei beziehungsweise ihrer Führung gegenüber missliebigen Abgeordneten und wurde bezeichnenderweise vor allem nach dem Ende des „Prager Frühlings“ in der Tschechoslowakei eingesetzt. ${ }^{20}$

\section{Funktionen der Volkskammer im realexistierenden Sozialismus}

Wenn es sich bei der Volkskammer dezidiert um kein „Parlament“ handelte, inwiefern ist es dann möglich, seinen Wandel zum Parlament zu untersuchen? Einerseits ist mit Heinrich Oberreuter festzuhalten, dass es im Kontext politischer Systeme, die den Deutungsmustern des Marxismus-Leninismus unterliegen, wenig sinnvoll ist, von „Parlamentarismus “ zu sprechen ${ }^{21}$, denn Vertretungskörperschaften in liberal-demokratischen und so genannten volksdemokratischen Systemen folgen völlig unterschiedlichen Leitideen. ${ }^{22}$ Andererseits wirbt Werner J. Patzelt für einen breiten Parlamentarismusbegriff, der alle Erscheinungsformen von Vertretungskörperschaften umfasst, auch wenn diese nicht von liberal-demokratischen Prinzipien geprägt sind. ${ }^{23}$ Dies ist erstens möglich, weil solche Vertretungskörperschaften einige Funktionen gemeinsam haben: So haben sie stets eine Repräsentationsfunktion ${ }^{24}$ und eine Kommunikationsfunktion ${ }^{25}$ zu erfüllen, wobei aber selbstverständlich nicht übersehen werden darf, dass die Inhalte dieser Funktionen entscheidend von den Werteordnungen der betreffenden politischen Systeme geprägt wer-

16 Vgl. dazu Peter Joachim Lapp, a.a.O. (Fn. 3), S. 56 - 63.

17 Vgl. Herwig Roggemann, Abgeordnetenrotation und Wähleraufträge in unterschiedlichen politischen Systemen. Ansätze zu einem verfassungspolitischen Vergleich, in: Ralf Rytlewski (Hrsg.), Politik und Gesellschaft in sozialistischen Ländern. Ergebnisse und Probleme der SozialistischeLänder-Forschung, Opladen 1989, S. 177 - 197.

18 Siegfried Lammich, a.a.O. (Fn. 3), S. 82.

19 Vgl. Peter Joachim Lapp, a.a.O. (Fn. 3), S. 63 f.; Siegfried Mampel, Die sozialistische Verfassung der Deutschen Demokratischen Republik. Kommentar, Frankfurt am Main 1981, S. 964 (Art. 57, Rn. 9).

20 Vgl. Siegfried Lammich, a.a.O. (Fn. 3), S. 83 f.

21 Vgl. Heinrich Oberreuter, a.a.O. (Fn. 2), S. 285.

22 Das unterstreicht auch der Marxismus-Leninismus, welcher den Begriff „Parlamentarismus“ für seinen Herrschaftsbereich ablehnte. Vgl. „Parlamentarismus“, in: Getrud Schütz u.a., a.a.O. (Fn. 6).

23 Vgl. Werner J. Patzelt, Die Volkskammer als Gegenstand vergleichender Parlamentarismusforschung, in: ders. / Roland Schirmer, a.a.O. (Fn. 2), S. 13 - 25, S. 14 f.

24 Vgl. Hanna Fenichel Pitkin, The Concept of Representation, Berkeley 1967, S. 209 f.

25 Vgl. zur vielfältigen Ausfüllung nicht nur durch Parlamentarier liberal-demokratischer Parlamente: Uwe Kranenpohl, Responsivität und Repräsentanten. Eine Analyse der Ergebnisse der empirischen Repräsentationsforschung, Magisterarbeit, Universität Passau 1991. 
den. ${ }^{26}$ Zweitens stellen solche Vertretungskörperschaften eine Infrastruktur für institutionelle Evolution dar, so wie sich bekanntlich auch die „Mutter der Parlamente“ in Westminster erst allmählich zu einem liberalen und noch viel später zu einem demokratischen Parlament wandelte. Ein eben solcher Wandlungsprozess ist auch Gegenstand dieser Untersuchung. Drittens ist festzuhalten, dass die Funktionen liberal-demokratischer Parlamente die der Volkskammer zugeschriebenen Funktionen gut erfassen, sofern die differierende „Wertefolie“ des Marxismus-Leninismus berücksichtigt wird.

Dass sich aus den beiden Positionen kein Widerspruch ergeben muss, zeigt sich exemplarisch an einer Untersuchung von Oberreuter, der die sozialistische Vertretungskörperschaft Volkskammer mithilfe der klassischen Parlamentsfunktionen analysierte. ${ }^{27}$ Tatsächlich wurden der Volkskammer - unter Berücksichtigung der spezifischen marxistisch-leninistischen „Wertefolie“ - Funktionen zugeschrieben, die jenen liberal-demokratischer Parlamente entsprechen. ${ }^{28}$

(1) Die Volkskammer bestellte gemäß Art. 50 DDR-V die Mitglieder von Staatsrat, Ministerrat und Oberstem Gericht, den Generalstaatsanwalt sowie den Vorsitzenden des Nationalen Verteidigungsrates und konnte diese jederzeit abberufen (Wahl- und Kreationsfunktion).

(2) Sie war gemäß Art. 48 II DDR-V „das einzige verfassungs- und gesetzgebende Organ" (Gesetzgebungsfunktion).

(3) Als sozialistische Vertretungskörperschaft hatte sie auch die Funktion, die Gesellschaft zu repräsentieren, was die Fraktionen ausgewählter Massenorganisationen deutlich machten. Als Verkörperung der „politische[n] Macht, die unter Führung der marxistischleninistischen Partei von der Arbeiterklasse im Bündnis mit den Genossenschaftsbauern, der Intelligenz und den anderen werktätigen Schichten ausgeübt" wurde ${ }^{29}$, war die Repräsentationsfunktion der Volkskammer aber selbstverständlich ideologisch geprägt und bezog sich lediglich auf die „sozialistische Gesellschaft“.

(4) Insbesondere den Abgeordneten der Volkskammer wurde explizit eine Kommunikationsfunktion zugesprochen. Gemäß Art. 56 III und IV DDR-V hatten sie „enge Verbindung zu ihren Wählern“ zu halten und waren „verpflichtet, deren Vorschläge, Hinweise und Kritiken zu beachten und für eine gewissenhafte Behandlung Sorge zu tragen“, aber auch „den Bürgern die Politik des sozialistischen Staates“ zu erläutern, also an der sozialistischen Umgestaltung - beziehungsweise Indoktrination - der Gesellschaft aktiv mitzuwirken. ${ }^{30}$

(5) Außerdem beinhaltete der Charakter der Volkskammer als „oberstes Staatsorgan“ eine Kontrollfunktion gegenüber allen staatlichen Organen - aber wohlgemerkt nicht gegenüber der SED als führender gesellschaftlicher und politischer Kraft. Vielmehr sollte die Volkskammer die Partei bei der gesellschaftlichen Kontrolle unterstützen, denn „alle

26 Darauf weisen auch offizielle DDR-Publikationen hin, in denen die Volkskammer als ,sozialistisches Parlament" bezeichnet wird. Vgl. etwa Herbert Kelle / Tord Riemann, a.a.O. (Fn. 9), S. 10.

27 Vgl. Heinrich Oberreuter, Sozialistischer Parlamentarismus?, a.a.O. (Fn. 4), S. $234-244$.

28 Vgl. für eine Übersicht der in der Literatur postulierten Funktionskataloge: Peter Schindler, Datenhandbuch zur Geschichte des Deutschen Bundestages 1949 bis 1999, Baden-Baden 1999, S. $2834-2847$.

29 Stichwort „Volkskammer“, in: Gertrud Schütz u.a., a.a.O. (Fn. 6), S. 1048.

30 Art. 57 I DDR-V verpflichtete die Abgeordneten sogar zur regelmäßigen Durchführung von Sprechstunden. 
Bereiche des gesellschaftlichen Lebens - die Volkswirtschaft, die Wissenschaft, das soziale Leben, die Bildung, die Kultur, die Arbeits- und Lebensbedingungen, die Ordnung und Sicherheit und der Schutz des Landes - unterliegen ihrer [...] Kontrolle“31.

\section{Der Wandel der Parlamentsfunktionen der Volkskammer $1989 / 90$}

Im Winter 1989/90 begann die Volkskammer, diese Potentiale zur Wahrnehmung von Funktionen, wie sie Parlamenten in liberal-demokratischen politischen Systemen zukommen, für sich zu entdecken.

\subsection{Wahl- und Kreationsfunktion}

Am Beginn des parlamentarischen Transformationsprozesses stand ein Wandel in der Wahlfunktion - und zwar zunächst in der Form, dass sich nicht mehr jeder Abgeordnete aufgefordert sah, Vorschläge der SED einfach „abzunicken“, sondern auch Gegenstimmen aufkamen. Zwar konnte Präsident Horst Sindermann (SED) auf der 10. Sitzung der Volkskammer (24. Oktober 1989) bei den Abberufungen von Erich Honecker als Vorsitzender des Staatsrates und des Nationalen Verteidigungsrates sowie von Günter Mittag als Stellvertretender Staatsratsvorsitzender noch die übliche Einheitlichkeit realsozialistischer „Abstimmungsmaschinen“ feststellen. Bei der ohne Aussprache erfolgten Wahl von Egon Krenz zum neuen Staatsratsvorsitzenden war Sindermann aber offenkundig überfordert, als er die Zahl der Gegenstimmen verkünden wollte: Nicht 22 Abgeordnete, wie er zunächst meinte, sondern 26 von 500 Mitgliedern der Volkskammer sprachen sich gegen Krenz aus, dazu kamen noch eine gleich große Zahl von Stimmenthaltungen. Auch beim sich anschließenden Wahlgang für den Vorsitz im Nationalen Verteidigungsrat konnte Krenz nicht die gewohnte Einstimmigkeit erzielen: Es gab acht Gegenstimmen und 17 Enthaltungen, wie Sindermann schon deutlich routinierter feststellte. ${ }^{32}$

Bei der 11. Tagung (13. November 1989) war Sindermann bereits von seinem Amt als Volkskammerpräsident zurückgetreten, weshalb der 92-jährige Alterspräsident Hans Jedretzky (FDGB - SED) zunächst die Neuwahl eines Parlamentsvorsitzenden durchführen musste. Die Fraktionen aller vier Blockparteien sowie der KB unterbreiteten je einen Personalvorschlag, die SED - obwohl stärkste Fraktion - hatte darauf verzichtet. Im ersten Wahlgang der geheimen Abstimmung erreichte keiner der Kandidaten die erforderliche absolute Mehrheit. Im zweiten Wahlgang der beiden am besten platzierten setzte sich der DBD-Kandidat Maleuda durch. Erste Amtshandlung des neuen Volkskammerpräsidenten war die Neuwahl der weiteren Präsidiumsmitglieder, da das alte Führungsgremium mit Sindermann zurückgetreten war. Auf gemeinsamen Antrag aller Fraktionen entsandte - wie auch zuvor immer praktiziert - jede Fraktion ein Präsidiumsmitglied. Die Wahl erfolgte bei einer Gegenstimme und drei Stimmenthaltungen. ${ }^{33}$

31 Stichwort „Volkskammer“, in: Gertrud Schütz u.a., a.a.O. (Fn. 6), S. 1049.

$32 \mathrm{StB} 9 / 222 \mathrm{f}$.

33 StB 9/230 - 232. Die Wahl des SED-Fraktionsvorsitzenden Werner Jarowinsky zum einzigen Stellvertreter Maleudas war im Ergebnis faktisch irrelevant, da der Präsident die Tagungen der Volkskammer ausnahmslos selbst leitete. 
Bis zum Ende der Wahlperiode folgten die Wahlen dann diesem Muster: Erforderliche Abberufungen erfolgten einstimmig, Neuwahlen in hochgradigem Einvernehmen bei wenigen Gegenstimmen und Enthaltungen. ${ }^{34}$ Lediglich bei der Wahl von Hans-Jürgen Joseph (SED) auf die politisch brisante Position des Generalstaatsanwalts kam es zu zwölf Gegenstimmen und 31 Enthaltungen. ${ }^{35}$

Für die Kreationsfunktion von besonderer Bedeutung ist die Regierungsbildung - insbesondere wenn, wie in der DDR, der Ministerrat lediglich als ausführendes Organ der Volkskammer fungieren soll. Die Umbildung der Regierung vollzog sich 1989/90 in mehreren Schritten: Zunächst wurde am Ende der 11. Tagung Hans Modrow (SED) auf Vorschlag seiner Partei bei lediglich einer Gegenstimme zum neuen Vorsitzenden des Ministerrats gewählt und ihm der Auftrag zur Regierungsbildung erteilt. ${ }^{36}$ Auf der folgenden Tagung (17./18. November 1989) gab Modrow eine Regierungserklärung ab und stellte sein Kabinett vor, das sich wie bis dahin üblich aus Vertretern aller in der Volkskammer vertretenen Parteien zusammensetzte. ${ }^{37}$ Die Volkskammer bestätigte die Regierung bei einer Gegenstimme und vier Enthaltungen. ${ }^{38}$ Bemerkenswert war in diesem Zusammenhang dreierlei: (1) Modrow, aber auch die Sprecher der anderen Parteien, titulierten die neue Ministerriege als „Koalition“ beziehungsweise „Koalitionsregierung“39 - eine Begrifflichkeit, die bis dahin in Zusammenhang mit der Konstituierung der DDR-Regierung undenkbar war und deutliche Anleihen bei den Verhältnissen in Ländern machte, die wenige Wochen zuvor noch als „Klassenfeinde“ galten. (2) Zwar stellte die SED immer noch mehr als die Hälfte der Minister, doch erhielten die anderen Parteien deutlich mehr und auch gewichtigere Ressorts. (3) In der Aussprache über die Regierungserklärung ${ }^{40}$ wurden unterschiedliche Auffassungen über Richtung, Umfang und Geschwindigkeit der Reformen zwischen SED und Massenorganisationen einerseits, LDPD und insbesondere CDU andererseits deutlich. Der CDU-Parteivorsitzende Lothar de Maizière sah sich in seinem Debattenbeitrag sogar genötigt zu erklären, die CDU begänne „den Wahlkampf heute noch nicht" ${ }^{* 1}$.

$\mathrm{Zu}$ einer denkwürdigen Umstrukturierung der Koalitionsregierung kam es auf der 16. Sitzung (5. Februar 1990), als sie durch die Aufnahme von acht Vertretern oppositioneller Gruppen zur „Regierung der nationalen Verantwortung “ 42 erweitert wurde. Die Abstimmung zeigte allerdings auch, dass die Erweiterung der Regierung um diese politischen Kräfte in der Volkskammer nicht auf ungeteilte Zustimmung stieß: Es gab 16 Gegenstimmen und 73 Enthaltungen. ${ }^{43}$

34 StB 9/271 f. (12. Tagung, 17./18. November 1989); StB 9/360, 9/369 f. (14. Tagung, 11./12. Januar 1990).

35 StB 9/370 (14. Tagung, 11./12. Januar 1990).

$36 \mathrm{StB} 9 / 264$.

37 StB 9/272 - 281.

38 StB $9 / 307$.

39 So etwa der SED-Fraktionsvorsitzende Jarowinsky noch vor der Wahl Modrows (StB 9/232; 11. Tagung, 13. November 1989)

40 StB $9 / 281-307$.

41 StB $9 / 287$.

42 StB 9/455 (Modrow).

$43 \mathrm{StB} 9 / 458$. 


\subsection{Kontrollfunktion}

Ab Mitte November ging die Volkskammer zügig daran, auch die in der Verfassung vorgesehene Kontrollfunktion gegenüber den ihr nominell unterstehenden staatlichen Organen wahrzunehmen. Besondere Kritik zog in der 11. Tagung (13. November 1989) zunächst der zurückgetretene Volkskammerpräsident Sindermann auf sich, der sich entgegen Art. 62 III DDR-V nach der 10. Sitzung drei Wochen lang der Forderung der Blockparteien verweigert hatte, eine weitere Plenarsitzung einzuberufen. ${ }^{44}$ Aber auch dem zurückgetretenen, von Willi Stoph (SED) geleiteten Ministerrat erging es im Rahmen der „Aussprache zur politischen Lage in der Deutschen Demokratischen Republik“ 45 nicht besser, in deren Rahmen erstmals Regierungsmitglieder vor einer republikweiten Öffentlichkeit „peinlichst“ befragt wurden. ${ }^{46}$ Während Stoph allerdings in erster Linie darauf verwies, die Regierung habe realiter dem ZK der SED unterstanden und deshalb kaum über eigenständige Gestaltungsspielräume verfügt, und ansonsten routiniert die geläufigen Floskeln kommunistischer Selbstkritik äußerte, ${ }^{47}$ bemühten sich Hochschulminister Hans-Joachim Böhme ${ }^{48}$, Finanzminister Ernst Höfner ${ }^{49}$ und der Vorsitzende der Plankommission Gerhard Schürer ${ }^{50}$ (alle SED) deutlich stärker, die Fragen der Abgeordneten zu beantworten. Den Höhepunkt dieser Regierungsbefragung stellte der denkwürdige Rechtfertigungsversuch des Ministers für Staatssicherheit Erich Mielke (SED) dar, der mit seinen Hinweisen, die Mitarbeiter seines Ministeriums hätten „einen außerordentlich hohen Kontakt zu allen werktätigen Menschen“ und er „liebe doch alle Menschen“ mehrmals Heiterkeit, ansonsten durch sein Statement aber vor allem Proteste auslöste. ${ }^{51}$

In der folgenden Zeit konzentrierte sich die Volkskammer in ihrer Kontrollfunktion zunächst darauf, die Vorkommnisse bis zur „Wende“ aufzuarbeiten. Zu diesem Zweck konstituierte sie in der 12. Tagung (17./18. November 1989) den „Zeitweiligen Ausschuss zur Überprüfung von Fällen des Amtsmissbrauchs, der Korruption, der persönlichen Bereicherung und anderer Handlungen, bei denen der Verdacht der Gesetzesverletzung besteht" -

44 Peter Joachim Lapp, a.a.O. (Fn. 2), S. 120. Vgl. zur Stimmungslage in Präsidium und Fraktionen: Herbert Kelle, Zeuge der Arbeit der Volkskammer über fast drei Jahrzehnte, in: Werner J. Patzelt I Roland Schirmer, a.a.O. (Fn. 2), S. $195-205$, S. 202 f.

45 StB 9/232 - 263.

46 In den Ausschüssen der Volkskammer sollen schon vor der Wende Kontrollaktivitäten eine gewisse Rolle gespielt haben (so Karl-Heinz Schulmeister, Ausschussarbeit am Beispiel des Kulturausschusses, in: Werner J. Patzelt / Roland Schirmer, a.a.O. (Fn. 2), S. 215 - 225). Allerdings betont auch er: „Zwar herrschte bei uns im Ausschuss für Kultur eine kritische Atmosphäre; Mängel und Schwierigkeiten wurden diskutiert. Aber in der öffentlichen Debatte - im Plenum der Volkskammer - fand diese Diskussion nicht statt“ (ebenda, S. 275). Sofern kontrolliert wurde, dominierte eindeutig die Leistungskontrolle, genuin politisch konnte die Kontrolltätigkeit nur werden, wenn Differenzen zwischen Mitgliedern des Ministerrats und der Parteiführung (Politbüro und ZKSekretäre) auftraten. Letztere nutzten ihre parlamentarischen Funktionen dann, um Druck auf die Regierungsmitglieder auszuüben. Vgl. dazu Roland Schirmer, Die Volkskammer - ein „stummes" Parlament? Die Volkskammer und ihre Abgeordneten im politischen System der DDR, in: Werner J. Patzelt / ders., a.a.O. (Fn. 2), S. $94-180$, S. 123 - 126.

$47 \mathrm{StB} 9 / 255,257 \mathrm{f}$.

$48 \mathrm{StB} 9 / 252 \mathrm{f}$.

$49 \mathrm{StB} 9 / 256 \mathrm{f}$.

50 StB $9 / 259-262$.

$51 \mathrm{StB} 9 / 262 \mathrm{f}$. 
also einen Untersuchungsausschuss. ${ }^{52}$ Das gleiche Ziel verfolgte auch der angeforderte Bericht des Generalstaatsanwaltes Günter Wendland (SED) bezüglich der Niederschlagung der Protestdemonstrationen zum 40. Jahrestag der Gründung der DDR am 7. Oktober, zu dem eine ganze Reihe kritischer Nachfragen gestellt wurden. ${ }^{53}$

Auf der 13. Sitzung (1. Dezember 1989) stand dann erstmals in der Wahlperiode der Punkt „Anfragen an die Regierung der DDR zu aktuellen Problemen“ auf der Tagesordnung der Volkskammer ${ }^{54}$, obwohl Art. 59 DDR-V ein entsprechendes Fragerecht für jeden Abgeordneten vorsah. ${ }^{55}$ Dabei kam es allerdings zu einigen Problemen: Wohl um jeden Eindruck von Manipulation zu vermeiden, verzichtete die Volkskammer auf Vorschlag von Maleuda auf schriftliche Wortmeldungen. ${ }^{56}$ Schon bald zeigte sich aber, dass dieses Verfahren nicht praktikabel war: „Es liegen sehr viele Wortmeldungen vor. Vielleicht können wir in etwa einen solchen Modus wählen, dass zunächst aus allen Fraktionen jeweils ein Abgeordneter zu Wort kommt [...]. "57 So verfuhr die Kammer auch, so dass zunächst je ein Vertreter der zehn Fraktionen die Möglichkeit zur Frage erhielt. In einer zweiten Runde erhielt dann je ein Abgeordneter von CDU, NDPD, FDGB, FDJ und KB nochmals die Möglichkeit einer Anfrage.

Ein anderes Problem ergab sich daraus, dass zumindest zu Beginn die Beiträge kaum den Charakter von Anfragen hatten: Klaus Herzog (FDJ) sprach als erster für seinen an den Rollstuhl gefesselten Fraktionskollegen Uwe Gajewski - der an diesem Tage erkrankt war und stellte gerade keine Anfrage, sondern gab ein behindertenpolitisches Statement ab. Präsident Maleuda verzichtete darauf, die „Anfrage“ vom Ministerrat beantworten zu lassen, und ging ohne Aufhebens zur nächsten Wortmeldung von Heinrich Toeplitz (CDU) über. ${ }^{58}$ Dieser verwies zunächst darauf, dass er vorgeschlagen habe, den Tagesordnungspunkt „Anfragen und Diskussion zu aktuellen Problemen“ zu nennen und warf dann drei „Fragen“ auf, die eher den Charakter von Debattenbeiträgen hatten und deren Beantwortung durch den angesprochenen Generalstaatsanwalt Wendland vergleichsweise knapp ausfiel. ${ }^{59}$ Am Ende des Tagesordnungspunktes - man sah sich außerstande, alle Wortmeldungen abzuarbeiten - mahnte Bernhard Mögling (NDPD) zu mehr Disziplin bei Parlamentariern wie Regierung: „Wenn wir Anfragen an die Regierung haben, dann können wir alle zu Wort kommen lassen [...], wenn sich diejenigen, die Fragen haben, kurz und präzise an die Regierung wenden und nicht Erklärungen abgeben. Zu den Ministern muss ich sagen: [...] Es geht nicht, dass vorbereitete halbe Regierungserklärungen von den einzelnen Ministern abgegeben werden."60 Allerdings stellte sich im weiteren Verlauf der Beratung eher das typische Wechselspiel parlamentarischer Anfragen ein: Die Fragen wurden etwas kürzer, und die Antworten der Regierung fielen in der Regel deutlich länger als die gestellten Fragen aus.

$52 \mathrm{StB}$ 9/307. Das eigenständige Institut eines Untersuchungsausschusses sahen weder DDR-Verfassung noch die Geschäftsordnung der Volkskammer vor.

53 StB 9/308-314.

54 StB 9/328 - 340.

55 Vgl. auch $\$ 12$ der Geschäftsordnung der Volkskammer.

$56 \mathrm{StB} 9 / 329$.

57 StB 9/333 (Maleuda).

$58 \mathrm{StB} 9 / 328 \mathrm{f}$.

$59 \mathrm{StB} 9 / 329 \mathrm{f}$.

$60 \mathrm{StB} 9 / 343$. 
Der Antrag auf Abschluss des Tagesordnungspunktes fand insbesondere deshalb eine Mehrheit $^{61}$, weil auch der darauf folgende die Volkskammer in ihrer Kontrollfunktion ansprach: Toeplitz gab als Vorsitzender des Untersuchungsausschusses nämlich einen ersten Zwischenbericht ab, der teilweise Heiterkeit, vor allem aber immer wieder Unmut auslöste. ${ }^{62}$ Aus den Befunden resultierten zahlreiche weitere Anfragen an die Regierung, deren Beantwortung offenkundig nicht immer als befriedigend empfunden wurde. ${ }^{63}$

Nach der Jahreswende machte die Volkskammer auf ihrer 14. Sitzung (11./12. Januar 1990) zunächst Anstalten, die Regierungstätigkeit intensiver zu kontrollieren. Zu Beginn der Sitzung nahm sie eine Regierungserklärung Modrows entgegen und debattierte am folgenden Tag mehrere Stunden darüber. ${ }^{64}$ Um die mitlaufende Kontrolle - und sicher auch die Gesetzgebungsarbeit - zu intensivieren, setzte sie zudem mit den Ausschüssen für Wissenschaft und Technik, für Umweltfragen sowie für Frauenfragen drei weitere Fachausschüsse ein. ${ }^{65}$

Die 15. Tagung (29. Januar 1990) stand dann schon ganz im Banne der Entwicklungen am Zentralen Runden Tisch. ${ }^{66}$ Gleich zu Beginn wies Modrow in einer weiteren Regierungserklärung auf die sich nochmals verschärfende Krisensituation in der gesamten Republik hin und kündigte sowohl die nochmalige Vorverlegung der Volkskammerwahlen auf den 8. März als auch die Bildung der „Regierung der nationalen Verantwortung“ unter Einschluss der am Runden Tisch vertretenen Oppositionsgruppen an. Deutlich wurde in diesem Zusammenhang, dass der Volkskammer - nicht zuletzt aufgrund ihrer mangelnden Legitimation durch die Bürger der DDR - die Initiative zunehmend zugunsten des Runden Tischs in seiner Funktion als „Ersatzparlament" entglitt ${ }^{67}:$ Zwar zogen sich die Fraktionen im Anschluss an die Erklärung Modrows zur Beratung zurück, eine Aussprache fand bezeichnenderweise aber nicht statt. ${ }^{68}$ Allerdings legte - wie in der Tagesordnung vorgesehen - der Vorsitzende der Wirtschaftskommission, Gerhard Schürer, über die Lage der Volkswirtschaft einen Bericht vor, an den sich eine längere Aussprache anschloss. ${ }^{69}$ Die 17.

61 Wie groß das Bedürfnis, weitere Fragen zu stellen, trotzdem war, verdeutlichen 110 Gegenstimmen und 15 Enthaltungen (StB 9/343).

62 StB 9/343-346.

$63 \mathrm{StB} 9 / 346-353$.

64 StB 9/361 - 369, $390-408$.

65 StB 9/384. Bereits seit 1986 bestanden Fachausschüsse für Auswärtige Angelegenheiten, Nationale Verteidigung, Verfassung und Recht, Industrie, Bauwesen und Verkehr, Landwirtschaft, Forstwirtschaft und Nahrungsgüterwirtschaft, Handel und Versorgung, Haushalt und Finanzen, Arbeit und Sozialpolitik, Gesundheitswesen, Volksbildung, Kultur sowie Jugend. Daneben bestanden noch Ausschüsse für Geschäftsordnung, Mandatsprüfung sowie Eingaben der Bürger.

66 Vgl. zum Zentralen Runden Tisch: Uwe Thaysen, Der Runde Tisch. Oder: Wer war das Volk?, in: ZParl, 21. Jg. (1990), H. 1, S. 71 - 100 und H. 2, S. 257 - 308; ders., Der Runde Tisch. Oder: Wo blieb das Volk. Der Weg der DDR in die Demokratie, Opladen 1990; ders., Der Zentrale Runde Tisch der DDR. Leistungen und Legenden, in: Karl Wilhelm Fricke / Hans Lechner / ders., Errungenschaften und Legenden. Runder Tisch, Willkürherrschaft und Kommandowirtschaft im DDR-Sozialismus, Melle 1990, S. 55 - 79; ders. (Hrsg.), Der Zentrale Runde Tisch der DDR. Wortprotokoll und Dokumente, 5 Bände, Wiesbaden 2000. Vgl. außerdem Klemens Semtner, Der Runde Tisch in der DDR, München 1992.

67 Deutlich wurde dies auch am Beschluss, dass freiwerdende Mandate der örtlichen Staatsorgane mit Vertretern oppositioneller Gruppen besetzt werden konnten (StB 9/442 - 445).

$68 \mathrm{StB} 9 / 422 \mathrm{f}$.

$69 \mathrm{StB}$ 9/445 - 452. Offengebliebene Fragen wurden dann allerdings erst auf der übernächsten 17. Tagung (20./21. Februar 1990) beantwortet (StB 9/510 - 514). 
Sitzung (20./21. Februar 1990) wurde trotz der Regierungserklärung zum Treffen mit Bundeskanzler Helmut Kohl und der am folgenden Tag durchgeführten großen Debatte zur deutschen Frage bereits deutlich durch die bevorstehende Auflösung der Volkskammer geprägt und diente nur noch bedingt der Kontrolle der Regierungsarbeit. ${ }^{70}$

Daher blieb auch nach dem Jahreswechsel die Aufarbeitung der SED-Diktatur ein deutlicher Schwerpunkt der Kontrolltätigkeit. So zeigten sich die Abgeordneten auf der 14. Tagung (11./12. Januar 1990) sowohl mit den Ausführungen des Präsidenten des Obersten Gerichts Günter Sarge (SED) zur Rehabilitierung als auch mit Auskünften des Stellvertreters des Generalstaatsanwalts Harri Harrland zu den Ermittlungen gegen ehemalige Funktionäre wegen Regierungskriminalität höchst unzufrieden. ${ }^{71}$ Vehement trat das Plenum daher auch Versuchen entgegen, die Behandlung der brisanten Frage nach dem Zugriff der Staatsanwaltschaft auf die Akten der Staatssicherheit auf die folgende Sitzung zu verschieben. ${ }^{72}$ Die Ausführungen des Abgeordneten Werner Kalweit (SED) geben die Stimmung gut wieder: „Es ist nicht mehr zu ertragen, wie in diesem Haus und anderswo seit Monaten durch verschiedene Personen der Eindruck der Vertuschung erweckt wird oder tatsächlich vertuscht wird, indem anstatt auf konkrete Fragen zu antworten, die Verantwortung stets den Vorgängern oder anderen Institutionen zugeschoben wird. “73

Mögling bezichtigte Harrland sogar, hinsichtlich angeblicher Ermittlungsprobleme bewusst die Unwahrheit gesagt zu haben. ${ }^{74}$ Konsequenterweise beschloss die Volkskammer, den Staatsrat zu beauftragen, gemäß Art. 71 I DDR-V Disziplinarverfahren gegen Sarge und Harrland einzuleiten und damit eine Enthebung von ihren Ämtern zu ermöglichen. ${ }^{75}$ Der neue Generalstaatsanwalt Joseph erstattete auf der folgenden 15. Tagung (29. Januar 1990) dann detailliert Bericht und beantwortete auch die Anfragen der Abgeordneten umfassend. ${ }^{76}$ Schließlich nahm die Volkskammer auf ihrer letzten Tagung (6./7. März 1990) noch den Bericht des Untersuchungsausschusses ohne Aussprache einstimmig zur Kenntnis. ${ }^{77}$

\subsection{Gesetzgebungsfunktion}

Obwohl die Volkskammer gemäß Art. 49 I DDR-V über ihre Gesetzgebungstätigkeit die Entwicklung der DDR für jedermann verbindlich gestalten sollte, war die Kammer auf diesem Felde kaum aktiv (vgl. Tabelle 1). Dabei ist insbesondere zu beachten, dass die Zahl der verabschiedeten Vertragsgesetze mit der internationalen Anerkennung der DDR in den 1970er und 80er Jahren deutlich anstieg, die Zahl der „echten“ Gesetze aber kontinuierlich abnahm. Zuletzt verabschiedeten die Abgeordneten in den 1980er Jahren nur noch etwa

70 StB $9 / 471-474,496-509$.

71 StB 9/412- 418 .

72 StB 9/414.

73 StB 9/416.

$74 \mathrm{StB} 9 / 417$.

75 Dem Antrag gegen Harrland wurde bei 7 Enthaltungen zugestimmt, jenem gegen Sarge bei 26 Gegenstimmen und 45 Enthaltungen (StB 9/417). Sarge kam einer Amtsenthebung durch seinen Rücktritt am 15. Januar 1990 zuvor (StB 9/424).

76 StB 9/438- 442 .

77 StB 9/542 f. 
drei dieser Gesetze pro Jahr. Angesichts des Primats der SED handelte es sich dabei nur um eine reine Formalität, die keinerlei inhaltliche „Arbeit am Gesetz“ bedeutete; doch ist es bezeichnend, dass das Politbüro sogar auf die Einhaltung der Form zunehmend verzichtete. ${ }^{78}$ Neben der formalen Verabschiedung von Gesetzen konnte die Volkskammer auch über Beschlüsse norm(er)setzend tätig werden: „Unter einem Beschluss im Sinne des Art. 49 I ist die konkrete Entscheidung einer speziellen Frage oder eines Bündels von solchen zu verstehen. [...] Beschlüsse [enthalten] in der Regel sachlich und zeitlich begrenzte Maßnahmen, die sich aus der generellen Verantwortung der beschließenden Organe ergeben."79 Dabei konnten diese Beschlüsse durchaus weitreichende legislative Folgen haben, indem durch sie etwa Gesetze aufgehoben oder die Wahlperiode örtlicher Volksvertretungen verlängert werden konnten.

\begin{tabular}{|l|c|c|c|c|}
\hline \multicolumn{6}{|l|}{ Tabelle 1: Gesetzgebungstätigkeit der Volkskammer nach Dekaden } \\
\hline & Vertragsgesetze & Plangesetze & „echte“ Gesetze & insgesamt \\
\hline $1950-59$ & 22 & 19 & 119 & 160 \\
\hline $1960-69$ & 19 & 16 & 74 & 109 \\
\hline 1970-79 & 51 & 22 & 53 & 126 \\
\hline 1980-89 (bis 10. Tagung) & 60 & 20 & 30 & 110 \\
\hline
\end{tabular}

Quelle: Georg Brunner, Das Staatsrecht der Deutschen Demokratischen Republik, in: Josef Isensee / Paul Kirchhof (Hrsg.), Handbuch des Staatsrechts der Bundesrepublik Deutschland, Bd. 1, Heidelberg 2003, S. $531-596$, S. 563, Fn. 117.

Im Vergleich zu den Zuständen in den letzten Jahren vor der Wende entfaltete die Volkskammer zwischen Dezember 1989 und März 1990 eine beeindruckende Aktivität, wie Tabelle 2 verdeutlicht. Diese Gesetzgebungstätigkeit - für die auch die aufgeführten einschlägigen Beschlüsse zu berücksichtigen sind - begann auf der 13. Tagung (1. Dezember 1989) mit der Änderung von Art. 1 I DDR-V. Seit 1974 hatte dieser folgenden Wortlaut: „Die Deutsche Demokratische Republik ist ein sozialistischer Staat der Arbeiter und Bauern. Sie ist die politische Organisation der Werktätigen in Stadt und Land unter Führung der Arbeiterklasse und ihrer marxistisch-leninistischen Partei."

Auf Antrag aller Fraktionen wurde beschlossen, den Führungsanspruch der „Arbeiterklasse und ihrer marxistisch-leninistischen Partei“ zu tilgen. Ein weitergehender Antrag der CDU-Fraktion - vertreten durch die Abgeordnete Christa Waldmann-Hojer -, auch die hervorgehobene Rolle der Arbeiter und Bauern zu streichen, fand dagegen nur die Unterstützung von 112 Abgeordneten und damit keine Mehrheit. ${ }^{80}$

Daran anschließend erfolgte die Erste Lesung des vom Ministerrat vorgelegten Reisegesetzes. Die intensiv geführte Debatte, an der sich insgesamt elf Abgeordnete aus acht Fraktionen beteiligten, war vor allem durch zahlreiche Fragen an Innenminister Lothar Ahrendt und Finanzministerin Uta Nickel (beide SED) gekennzeichnet. Sie konzentrierte sich nach der erfolgten Maueröffnung vor allem auf das Problem, ob angesichts der wirtschaftlichen

78 Vgl. Peter Joachim Lapp, a.a.O. (Fn. 3), S. 210 - 222; Roland Schirmer, a.a.O. (Fn. 46), S. $135-147$.

79 Siegfried Mampel, a.a.O. (Fn. 19), S. 921 (Art. 49, Rn. 5), dort auch zahlreiche Beispiele.

80 Bemerkenswert ist dabei, dass auch bei Annahme des Antrags die DDR weiterhin „ein sozialistischer Staat" und „die politische Organisation der Werktätigen" geblieben wäre. 


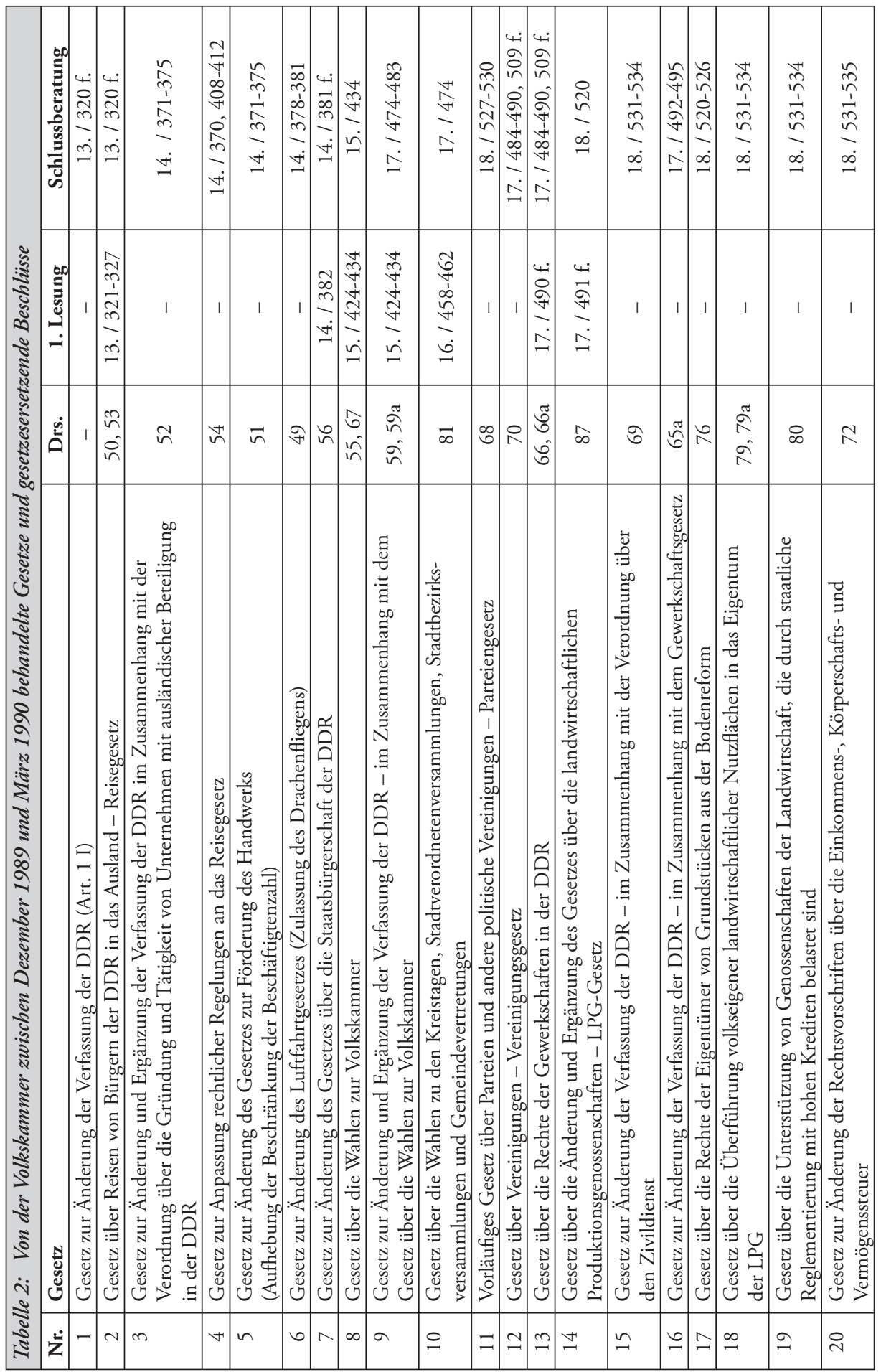


Krise ausreichend Devisen für die Finanzierung von Auslandsreisen der DDR-Bürger verfügbar seien. ${ }^{81}$

Die folgende 14. Tagung (11./12. Januar 1990) war durch die Beratung und Verabschiedung einer ganzen Reihe von Gesetzen gekennzeichnet, die alle von großer symbolischer Bedeutung waren:

(1) Das bereits beratene Reisegesetz wurde in Verbindung mit dem Gesetz zur Anpassung rechtlicher Regelungen an das Reisegesetz verabschiedet.

(2) Durch Änderung von Art. 12 DDR-V und die Einfügung des Art. 14a DDR-V wurde die Möglichkeit ausländischer Unternehmensbeteiligungen eröffnet. Dem Ziel, ideologische Gängelung im Bereich des Wirtschaftslebens zu beseitigen, diente auch die Änderung des Gesetzes zur Förderung des Handwerks, mit dem bislang bestehende Beschränkungen der Beschäftigtenzahl von Betrieben aufgehoben wurden.

(3) Ohne Debatte verabschiedete die Volkskammer eine Änderung des Luftfahrtgesetzes, durch die das - wegen der Gefahr der „Republikflucht“ - verbotene Drachenfliegen legalisiert wurde. ${ }^{82}$

(4) In Erster Lesung berieten die Abgeordneten über Änderungen des Staatsbürgerschaftsgesetzes, mit denen die Möglichkeit zur „Ausbürgerung“ beseitigt werden sollte ${ }^{83}$, sowie ein Zivildienstgesetz.

(5) Schließlich fasste die Volkskammer auch noch einen Beschluss zur Verkürzung ihrer Wahlperiode; die Neuwahl sollte am 6. Mai 1990 stattfinden.

Auch im Bereich der Gesetzgebung ging die Initiative aber zunehmend von der Volkskammer an den Zentralen Runden Tisch über, wie sich schon auf der 15. Tagung (29. Januar 1990) zeigte und was einigen Unwillen bei den Abgeordneten auslöste. ${ }^{84}$ Die am Runden Tisch getroffene Vereinbarung, die Wahlen nochmals auf den 18. März 1990 vorzuziehen, setzte das Parlament erheblich unter Druck. Zwar berieten die Abgeordneten auf dieser Tagung noch in Erster Lesung das Gesetz über die Wahlen zur Volkskammer sowie die in diesem Zusammenhang erforderlichen Verfassungsänderungen und verabschiedete auch das Gesetz zur Änderung des Staatsbürgerschaftsgesetzes. Die Gesetzgebungsarbeit der folgenden Sitzungen war aber vor allem dadurch geprägt, angesichts des schnell näher rückenden Wahltermins unbedingt erforderliche Regelungen zu erlassen, um die Wahl ordnungsgemäß durchführen beziehungsweise den ökonomischen Umstrukturierungsprozess fortsetzen zu können. Auch wegen dieses Zeitdrucks arbeitete die Volkskammer intensiv mit Beschlüssen gemäß Art. 49 DDR-V - so etwa beim Beschluss über die Gewährung von Meinungs-, Informations- und Medienfreiheit ${ }^{85}$ oder zu Aktivitäten der Partei „Die Republikaner“ ${ }^{86}$

Allerdings ist festzustellen, dass Gesetzesinitiativen aus der Mitte des Hauses auch in den Monaten nach der Wende die absolute Ausnahme waren. Lediglich der FDGB machte von seinem Recht gemäß Art. 65 I DDR-V Gebrauch und brachte von sich aus das Gesetz über die Rechte der Gewerkschaften in der DDR ein. Auch die DBD war mit einer Gesetzesini-

81 Dies verdeutlicht auch das intensiver parlamentarischer Gesetzgebungsarbeit innewohnende Moment „mitlaufender Kontrolle“.

82 Vgl. die Ausführungen von Verkehrsminister Heinrich Scholz (SED).

83 Vgl. die Ausführungen von Innenminister Ahrendt.

84 Vgl. 9/430 f. (Eva Rohmann, DFD; Johannes Zillig, CDU).

85 StB 9/434 - 438 (15. Tagung, 29. Januar 1990), 462 f. (16. Tagung, 5. Februar 1990).

86 StB 9/465 - 467 (16. Tagung, 5. Februar 1990). 
tiative über die Überführung volkseigener landwirtschaftlicher Nutzflächen in das Eigentum der LPG erfolgreich.

Insgesamt dominierte aber insbesondere in Fragen der Wirtschaftspolitik und Wirtschaftsverfassung sehr deutlich der Ministerrat, auch wenn in Einzelfällen seine Gesetzesinitiativen deutlich verändert wurden. ${ }^{87}$ Allerdings ist anhand der Plenarprotokolle nicht zu erschließen, wie intensiv die in den Ausschussberatungen erfolgten Veränderungen tatsächlich auf die Abgeordneten zurückgehen oder ob durch die Regierung „nachgebessert“ wurde. Aufklärung können nur die Ausschussdokumente geben, deren Edition durch die Kommission für die Geschichte des Parlamentarismus und der politischen Parteien in Aussicht steht. Offensichtlich wurde aber die Wahlgesetzgebung sehr deutlich durch den zeitweiligen Ausschuss geprägt.

Zudem führte die ursprüngliche sozialistische Konzeption der Volkskammer als eines „Feierabendparlaments der Werktätigen“ zu Problemen. Da die Abgeordneten in ihren Arbeitskollektiven verbleiben sollten, kam es mit der Intensivierung der parlamentarischen Tätigkeit zu tiefgreifenden Schwierigkeiten bei der Vereinbarkeit von Beruf und Mandat. ${ }^{88}$ Auch aus diesem Grund war wohl nicht immer sicher, ob die Volkskammer bei der Verabschiedung von Verfassungsänderungen beschlussfähig sein würde. ${ }^{89}$

Gerade bei der Wahrnehmung der Gesetzgebungsfunktion wird in der Gesamtbetrachtung die mangelnde demokratische Legitimation der Volkskammer und ihrer Abgeordneten deutlich. Spätestens mit der Etablierung des Zentralen Runden Tischs bestand ein konkurrierendes quasi-parlamentarisches Gremium, das überzeugender legitimiert war.

\subsection{Kommunikationsfunktion}

Durch die Wende veränderte sich auch die Kommunikationsfunktion der Volkskammer und ihrer Abgeordneten. Deren primäre Aufgabe war es im sozialistischen Parlamentarismus, die Entscheidungen der Partei und der von ihr angeleiteten Staatsorgane der Bevölkerung zu erklären; nur in homöopathischer Dosierung konnte sie dagegen darin bestehen, deren Bedürfnisse, Interessen und Anregungen dem politischen Entscheidungszentrum zu übermitteln. ${ }^{90}$ Nunmehr gewann aber gerade diese Funktion an Bedeutung - auch wenn der Abgeordnete Lutz Ahnfeld (FDJ - SED) auf der 11. Tagung (13. November 1989) nochmals ausdrücklich darauf verwies, dass es eine Verfassungsaufgabe der Abgeordneten sei, „die Politik des Staates zu erläutern“91.

Institutionell zeigte sich dies darin, dass erstmals Plenardebatten live im DDR-Fernsehen übertragen wurden ${ }^{92}$ - und wohl auch erstmals auf größeres öffentliches Interesse

87 So etwa zentrale Passagen des Reisegesetzes.

88 StB 9/444 (Verena Schlüsselburg, DBD; 15. Tagung, 29. Januar 1990).

89 Vgl. den entsprechenden Hinweis von Maleuda: StB 9/474 (17. Tagung, 20./21. Februar 1990).

90 Vgl. Roland Schirmer, a.a.O. (Fn. 46), S. 115 - 123.

$91 \mathrm{StB} 9 / 243$.

92 Vgl. Reiner Stein, Vom Fernsehen der DDR zur ARD. Die Entwicklung und Neuordnung des Rundfunkwesens in den Neuen Bundesländern, Marburg 2000, S. 56. Vgl. auch die OnlineDokumentation des Deutschen Rundfunkarchivs: „Die Volkskammer der DDR im Wandel“, http://1989.dra.de/themendossiers/politik/volkskammer.html (Abruf am 14. August 2009). 
stießen. Schon unmittelbar nach seiner Wahl zum Staatsratsvorsitzenden auf der 10. Sitzung (24. Oktober 1989) versprach Krenz mehr Transparenz: „Zur lebendigen Arbeit der Volkskammer gehört, dass die Massenmedien die Bürger fundiert und aktuell über den Prozess der parlamentarischen Willensbildung informieren. Die Öffentlichkeit will über die Tätigkeit der Volkskammer, ihrer Ausschüsse und der Abgeordneten Bescheid wissen. Dem sollte mit einer umfassenden und qualifizierten Parlamentsberichterstattung Rechnung getragen werden. "93 Noch waren dies aber nur Ankündigungen, wie auch der damalige Leiter des Sekretariats der Volkskammer, Herbert Kelle (SED), rückblickend feststellt: „Diese Volkskammertagung wurde vom überwiegenden Teil der Abgeordneten und auch von der Öffentlichkeit als äußerst formal, ja als undemokratisch empfunden. Die Reden, deren Inhalt, die Art und Weise des Verfahrens zeigten, dass alles an den im Leben entstandenen Fragen und Konflikten weit vorbeiging. "94

Wenig später allerdings debattierten die Abgeordneten - durchaus kontrovers - über die „politische Lage in der Deutschen Demokratischen Republik“95 und fanden echte Aussprachen zu Regierungserklärungen statt. ${ }^{96}$ Mit dem Bedeutungsgewinn des Zentralen Runden Tisches verlor die Volkskammer aber auch in ihrer Debattenfunktion an Bedeutung. Die „Aussprache zur deutschen Frage“ auf der 17. Tagung (20./21. Februar 1990) stellte eine Ausnahme dar, fand aber schon im Schatten der Volkskammerwahlen statt.

Die Zahl der Eingaben an die Volkskammer und ihre Gremien explodierte ${ }^{97}$; so gab es allein „Tausende von Zuschriften und Unterschriftensammlungen“ zur Streichung des Führungsanspruchs der SED aus der Verfassung. ${ }^{98}$ Es wandten sich eine so große Anzahl von Bürgern mit „Zuschriften, Telegrammen und Anrufen“ an die Volksvertretung, dass Präsident Maleuda bereits auf der 13. Tagung (1. Dezember 1989) darauf hinwies, es sei „nicht möglich, diese Wortmeldungen einzeln zu beantworten“. Er sah sich sogar gezwungen, Kommunikationsanliegen der DDR-Bürger mit Verweis auf Art. 48 und 49 DDR-V an andere Stellen zu verweisen. ${ }^{99}$

Gemäß Art. 65 III DDR-V waren die Entwürfe grundlegender Gesetze einer „Volksaussprache“ zu unterwerfen. ${ }^{100}$ Eine solche Aussprache führte die Volkskammer beim Wahlgesetz durch, an der sich, wie Paul Eberle (LDPD), der Vorsitzende des Zeitweiligen Ausschusses zur Ausarbeitung eines neuen Wahlgesetzes, ausführte, fast 16.000 Bürger beteiligten. ${ }^{101}$ Eine weitere fand auch zum Gewerkschaftsgesetz statt. ${ }^{102}$ An zahlreichen anderen Gesetzgebungsprojekten nahmen interessierte Bürger von sich aus mit Eingaben teil, so etwa beim Reisegesetz. ${ }^{103}$

93 StB 9/225.

94 Herbert Kelle, a.a.O. (Fn. 44), S. 202.

95 StB 9/232 - 263 (11. Tagung, 13. November 1989).

96 StB 9/281 - 306 (12. Tagung, 1. Dezember 1989); StB 390 - 408 (14. Tagung, 11./12. Januar 1990).

97 StB 9/306 f. (Maleuda; 12. Tagung, 17./18. November 1989).

98 StB 9/321 (Maleuda; 13. Tagung, 1. Dezember 1989).

99 Vgl. StB 9/328.

100 Vgl. Siegfried Mampel, a.a.O. (Fn. 19), S. 994 f. (Art. 65, Rn. 15).

101 StB 9/474 (17. Tagung, 20./21. Januar 1990).

102 StB 9/520 (Maleuda; 18. Tagung, 6./7. März 1990).

103 StB 9/372 (Innenminister Ahrendt; 14. Tagung, 11./12. Januar 1990). 
Zwar betonten in den Plenardebatten Vertreter aller Fraktionen, nach der Wende müssten die Abgeordneten der Volkskammer stärker als zuvor im Auftrag der DDR-Bürger handeln; besonders deutlich wurde diese Position aber durch die Massenorganisationen vertreten. Vor allem bei deren Abgeordneten zeigte sich eine Tendenz, die zuvor zumindest deklaratorisch formulierte Responsivitätsfunktion, „die Interessen der Werktätigen, ihre Meinung und ihre schöpferischen Ideen fundiert [zu] vertreten" ${ }^{104}$, nunmehr ernsthaft auszufüllen. ${ }^{105}$ Doch sie waren noch stärker als jene der SED und der Blockparteien mit dem Problem konfrontiert, die sich wandelnde Gesellschaft der DDR nur eingeschränkt repräsentieren zu können.

\subsection{Repräsentationsfunktion}

Im Zuge der Wende verloren auch FDGB, FDJ, DFD, KB und VdgB ihr bis dahin bestehendes Monopol zur Organisation gesellschaftlicher Anliegen und Interessen, das für die SED unerlässlich war, um diese Vereinigungen als gesellschaftliche „Transmissionsriemen“ nutzen zu können. Schon auf der 11. Tagung (13. November 1989) wurde deutlich, dass die Massenorganisationen damit auf ihre nur rudimentär ausgebildete Funktion der Interessenvertretung zurückgeworfen waren ${ }^{106}$, während die Parteien dagegen deutlich ein „allgemeinpolitisches Mandat" beanspruchten. So trat der Abgeordnete Manfred Scheler (VdgB - SED) der bereits aufkommenden Diskussion um die Rolle der Massenorganisationen entgegen und forderte auch eine künftige Vertretung im Parlament. ${ }^{107}$

Letztlich standen die Massenorganisationen aber auf verlorenem Posten und nahmen sich in den Plenardebatten merklich zurück (Tabelle 3). ${ }^{108}$ Dieser Entwicklung musste auch der FDGB bei der Neufassung des Gewerkschaftsgesetzes Tribut zollen, wie seine Vorsitzende Helga Mausch (NDPD) bei dessen Verabschiedung einräumte. ${ }^{109}$ Besonders offenkundig wurde der Bedeutungsverlust bei der erst seit 1986 in der Volkskammer - mit zudem nur 14 Abgeordneten - vertretenen VdgB, die sich fast ausschließlich auf die Artikulation der (vermeintlichen) Interessen der Genossenschaftsbauern beschränkte. Ein ähnliches Bild zeigte auch der DFD sowie der FDGB. ${ }^{110}$ Deutlich aktiver war vor allem die FDJ, die zwar auch versuchte, sich zur Vertreterin der Interessen der jüngeren Generation zu stilisieren ${ }^{111}$, deren Abgeordnete aber selbstverständlich zur „Kaderreserve“ der SED zählten. Mit diesem Selbstverständnis stand die FDJ-Fraktion den Fraktionen der Parteien natürlich näher als die anderen Massenorganisationen.

104 Stichwort „Abgeordneter“, in: Gertrud Schütz u.a., (Fn. 6), S. 11.

105 Vgl. StB 9/258. Vgl. auch 9/240 f. (Kimmel, FDGB - SED).

$106 \mathrm{StB} 9 / 240-248$.

107 StB 9/247.

108 Gezählt wurde jeweils die Anzahl der Abgeordneten, die sich an der Debatte eines Tagesordnungspunkts beteiligten, sowie Nichtparlamentarier, die für eine der Fraktionen sprachen. Nicht berücksichtigt wurden Beiträge von Regierungsmitgliedern.

109 StB 9/520 f. (18. Tagung, 6./7. März 1990).

110 Beim FDGB ist die Beratung des von ihm eingebrachten Gewerkschaftsgesetzes zu beachten.

111 Vgl. etwa StB 9/243 - 245 (Ahnfeld; 11. Tagung, 13. November 1989). 


\begin{tabular}{|l|c|c|c|c|c|c|c|c|c|c|}
\hline Tabelle 3: Anzahl der Debattenbeiträge der Fraktionen in der Volkskammer 1989/90 \\
\hline & \multicolumn{10}{|c|}{ Fraktionen (Mandate) } \\
\hline Tagung (Datum) & SED & DBD & CDU & LDPD & NDPD & FDGB & FDJ & DFD & KB & VdGB \\
$(127)$ & $(52)$ & $(52)$ & $(52)$ & $(52)$ & $(615)$ & $(37)$ & $(32)$ & $(21)$ & $(14)$ \\
\hline 11. (13.11.1989) & 6 & 2 & 3 & 2 & 5 & 3 & 5 & 2 & 3 & 1 \\
12. (17./18.11.1989) & 6 & 3 & 4 & 6 & 4 & 5 & 3 & 1 & 2 & 1 \\
13. (1.12.1989) & 3 & 3 & 6 & 4 & 6 & 4 & 5 & 2 & 4 & 1 \\
14. (11./12.1.1990) & 7 & 5 & 9 & 10 & 9 & 3 & 4 & 2 & 3 & 2 \\
15. (29.1.199) & 3 & 9 & 5 & 5 & 4 & 1 & 4 & 2 & 2 & 1 \\
16. (5.2.1990) & 2 & 1 & 4 & 1 & 1 & 1 & 2 & 2 & 0 & 0 \\
17. (20./21.2.1990) & 5 & 6 & 8 & 3 & 9 & 3 & 2 & 2 & 2 & 1 \\
18. (6./7.3.1990) & 8 & 5 & 6 & 3 & 3 & 3 & 4 & 2 & 2 & 0 \\
\hline Summe & 40 & 34 & 45 & 34 & 40 & 21 & 29 & 15 & 18 & 7 \\
\hline Quelle: Eigene Auszählung anhand der StB.
\end{tabular}

Allerdings bestanden auch unter den Blockparteien feine Unterschiede ${ }^{112}$ : Konnten sich CDU und LDPD zumindest noch auf ihre demokratischen Wurzeln in der unmittelbaren Nachkriegszeit beziehen, so waren DBD und NDPD 1948 als reine Satellitenparteien der SED gegründet worden. ${ }^{113}$ Um so deutlicher suchte sich die DBD als „authentische“ Vertreterin landwirtschaftlicher Interessen zu profilieren ${ }^{114}$, als deren Anspruch, die Genossenschaftsbauern gesellschaftlich zu erfassen, schon vor der Wende von der Massenorganisation $\mathrm{VdgB}$, aber auch von den anderen Blockparteien beeinträchtigt wurde. ${ }^{115}$ Noch bedrohter war die NDPD, die als Vertreterin von ursprünglich „national geprägten kleinbürgerlich-demokratischen Kräften "116 noch stärker als die DBD in Konkurrenz zu CDU und LDPD stand. Sowohl hinsichtlich der Reform des Wirtschaftssystems als auch der Überwindung der Teilung Deutschlands agierten diese aber glaubhafter: So beantragte etwa auf der 16. Tagung (5. Februar 1990) der CDU-Abgeordnete Dietmar Czok - und nicht etwa ein Vertreter der Nationaldemokraten - erfolgreich die Ansetzung einer Aussprache zur deutschen Frage. ${ }^{117}$

112 Vgl. zur Entwicklung der Blockparteien ab Herbst 1989: Peter Joachim Lapp, Ausverkauf. Das Ende der Blockparteien, Berlin 1998; Michael Richter, Zur Entwicklung der Ost-CDU im Herbst 1989, in: Historisch-Politische Mitteilungen, 1. Jg. (1994), S. 115 - 133; Siegfried Suckut, Vom Blocksystem zur Konkurrenz. Zum Wandel der ehemaligen Blockparteien in der DDR seit dem Herbst 1989, in: Ilse Spittmann / Gisela Helwig, Die DDR auf dem Weg zur Deutschen Einheit. Probleme - Perspektiven - offene Fragen. 23. Tagung zum Stand der DDRForschung in der Bundesrepu-blik Deutschland, 5. bis 8. Juni, Köln 1990, S. 128 - 138.

113 Vgl. Peter Joachim Lapp, Die „befreundeten Parteien“ der SED. Blockparteien heute, Köln 1988, S. $11-16$.

114 Vgl. dazu den Gesetzentwurf über die Überführung volkseigener landwirtschaftlicher Nutzflächen in das Eigentum der LPG (StB 9/531 - 534; 18. Tagung, 6./7. März 1990).

115 Vgl. Peter Joachim Lapp, Die Blockparteien im politischen System der DDR, Melle 1988, S. 45.

116 So die Satzung der NDPD von 1987, abgedruckt in: Peter Joachim Lapp, a.a.O. (Fn. 113), S. $215-224$, S. 215.

117 StB 9/456 f. Die Debatte fand auf der folgenden 17. Tagung (20./21. Februar 1990) statt (StB $9 / 496-509)$. 
Nach der Wende versuchten alle Fraktionen der Volkskammer, ihre Legitimation zu erhöhen, indem sie insgesamt 84 politisch belastete Abgeordnete (davon 49 der SED-Fraktion) durch das Plenum gemäß $\$ 47$ IV Wahlgesetz abberufen und durch Nachfolgekandidaten ersetzen ließen. ${ }^{118}$ Dass diese Praxis im Februar 1990 nicht mehr fortgeführt wurde, ist wohl kaum ein Signal für einen erfolgreichen Abschluss des beabsichtigten „Säuberungsprozesses“. Statt dessen ist einerseits in Rechnung zu stellen, dass durch die abermals verkürzte Wahlperiode ein weiterer Austausch der Abgeordneten für die letzten Sitzungen der Volkskammer nicht mehr sinnvoll erschien. Andererseits - und das ist wohl noch wesentlicher - sahen die DDR-Bürger ihre politischen Positionen am Zentralen Runden Tisch, an dem sowohl die alten als auch die neuen politischen Kräfte vertreten waren, besser repräsentiert als durch die Volkskammer und die Abgeordneten der (ehemaligen) Blockparteien und Massenorganisationen. ${ }^{119}$

Als weitere Maßnahme zur Erhöhung der Repräsentativität der Volkskammer kann die gemäß $\$ 5$ II GO mögliche Praxis angesehen werden, auch Nichtparlamentariern ein Rederecht einzuräumen. Das erste Mal war dies bereits auf der 12. Tagung (17./18. November 1989) der Fall, als in der Aussprache zur Regierungserklärung Modrows für die CDU-Fraktion der neugewählte Parteivorsitzende Lothar de Maizière sprach. ${ }^{120}$ Als Vertreter des Zentralen Runden Tischs erhielten auf der 15. Sitzung (29. Januar 1990) Wolfgang Ullmann, Konrad Weiß (beide Demokratie Jetzt) und Tatjana Böhm (Unabhängiger Frauenverband) das Wort. ${ }^{121}$ Vor ihrer Wahl in die "Regierung der nationalen Verantwortung “ standen Walter Romberg (SPD) und abermals Ullmann auf der 16. Tagung (5. Februar 1990) Rede und Antwort. ${ }^{122}$ Als Vertreterin des FDGB erläuterte Helga Mausch auf der 17. Sitzung (20./21. Oktober 1990) den Entwurf des Gewerkschaftsgesetzes, anschließend sprach Rainer Ortleb als LPD-Vorsitzender im Rahmen der Aussprache zur deutschen Frage. ${ }^{123}$ Auf der letzten Tagung am 6./7. März 1990 nahm abermals Mausch gemeinsam mit ihrem Vorstandskollegen Siegfried Sahr (SED) zum nochmals überarbeiteten Gesetzentwurf Stellung. ${ }^{124}$ Schließlich erhielt auch noch Roland Acker als Experte des Zeitweiligen Ausschusses zur Ausarbeitung eines neuen Wahlgesetzes beim letzten Treffen der Volkskammer das Wort für erläuternde Bemerkungen. ${ }^{125}$

118 StB 9/272 (12. Tagung, 17./18. November 1989); StB 9/319 f. (13. Tagung, 1. Dezember 1989); StB 360 f. (14. Tagung, 11./12. Januar 1990); StB 9/424 (15. Tagung, 29. Januar 1990). Vgl. zu den Zahlenangaben Herbert Kelle, a.a.O. (Fn. 44), S. 202.

119 Vgl. „Vor allem eine Krise der Legitimität“, in: Tagesspiegel vom 28. Februar 1990.

$120 \mathrm{StB} 9 / 287-289$.

$121 \mathrm{StB} 9 / 427-429,436 \mathrm{f}$.

$122 \mathrm{StB} 9 / 457 \mathrm{f}$.

123 StB 9/490 f., $497 \mathrm{f}$.

$124 \mathrm{StB} 9 / 520$ f., $524-526$.

125 StB 9/530. Bezeichnenderweise hatte Acker als Mitarbeiter am Institut für Theorie des Staates und des Rechts der Akademie der Wissenschaften noch 1988 als Mitautor die „demokratischen Prinzipien" des DDR-Wahlsystems verfochten. Vgl. Oswald Unger / Roland Acker / Inge Fiedler, Wahlsystem und Volksvertretungen der DDR, Berlin (Ost) 1988. 


\section{Fazit: Von der „sozialistischen Volksvertretung“ zum (fast) echten, kurzen Parlament}

Die Wochen zwischen Ende Oktober 1989 und Anfang März 1990 liefern Argumente zur Bestätigung der Einschätzung Patzelts, alle Vertretungskörperschaften als „Parlamente“ zu klassifizieren ${ }^{126}$, denn das Beispiel der Volkskammer zeigt, dass selbst ein faktisch nahezu machtloses Gremium in einer Regimekrise weitgehend in die Wahrnehmung der Parlamentsfunktionen eintreten - und diese zu einem gewissen Grad erfüllen - kann. Der Grund scheint vor allem darin zu liegen, dass eine „Volksvertretung“ - selbst wenn ihre Mitglieder unter der Suprematie einer „absolutistischen Integrationspartei“127 rekrutiert wurden - schon durch ihre Größe eine stärkere Heterogenität aufweist als ein Politbüro oder ein Zentralkomitee. Gleiches gilt in sozialer und politisch-programmatischer Hinsicht wohl auch gegenüber einem Parteitag der dominierenden Staatspartei: Verglichen mit der SED - und vor allem ihren Führungsgremien - als bisherigem Herrschaftszentrum verfügte die Volkskammer tatsächlich über eine höhere Legitimation und konnte die DDR-Gesellschaft besser repräsentieren. ${ }^{128}$ Mit dem faktischen und später auch staatsrechtlich nachvollzogenen Wegfall der Suprematie der Kommunistischen Partei entfiel gleichsam die "Wertefolie“, die es den Abgeordneten bis dahin nicht erlaubt hatte, die ihnen nominell auch im realexistierenden Sozialismus zu einem gewissen Grad zugeschriebenen Parlamentsfunktionen wahrzunehmen.

Allerdings - und das ist ganz wesentlich - ging mit der Krise der Parteidiktatur die „Macht“ nicht auf die Volkskammer als „oberste[s] staatliche[s] Machtorgan“ (Art. 48 I DDR-V) über. Statt dessen lag sie - um es mit einem vielfach kolportierten und treffenden Bild zu beschreiben - „auf der Straße“, das heißt im öffentlichen politischen Diskurs der Gesellschaft. Besser als durch die 1986 gewählte Volkskammer wurden die unterschiedlichen politischen Positionen der DDR-Bürger durch den Zentralen Runden Tisch repräsentiert, an dem nicht nur (moderate) Vertreter der alten Staatspartei, wandlungswillige (und zum Teil auch -fähige) ehemalige Blockparteien und sich im Umbruch befindende ehemalige Massenorganisationen saßen, sondern eben auch neue politische Parteien (fast) aller Couleur - vom christdemokratischen „Demokratischen Aufbruch“ bis zur „Vereinigten Linken“ -, zivilgesellschaftliche Organisationen in Form der Bürgerbewegungen und nicht zuletzt (als nicht stimmberechtigte Moderatoren) die Kirchen. Selbstverständlich waren auch die Mitglieder dieses „Quasi-Parlaments“ nicht von den Bürgern gewählt worden, aber unzweifelhaft repräsentierte es die DDR-Gesellschaft unvergleichlich besser als selbst

126 Vgl. Werner J. Patzelt, a.a.O. (Fn. 23), S. 14 f.

127 Sigmund Neumann, Die Parteien der Weimarer Republik, Stuttgart 1973 [1932], S. 107.

128 Vgl. dazu auch die Position von Georg Brunner, der mit Blick auf die reformfreudigsten Ostblockstaaten (Polen, Ungarn und die Sowjetunion) bemerkte: „Die Vorgänge im parlamentarischen Raum erinnern an die Endphase der absoluten Monarchie in Westeuropa, als die Allmacht des Monarchen - hic et nunc: der Parteiführung - durch eine zunehmend demokratischer werdende Repräsentativversammlung des Volkes immer mehr beschnitten und schließlich auf eine Reservemacht beschnitten wurde." Vgl. Georg Brunner, Ansätze zu einem „sozialistischen Parlamentarismus" im sowjetischen Hegemonialbereich, in: Ralf Rytlewski, a.a.O. (Fn. 17), S. 151 176, S. 175. Man beachte dabei aber, dass in diesen Systemen bereits eine gewisse Öffnung der Wahlverfahren stattgefunden hatte; ebenda, S. 163 - 166; S. $171 \mathrm{f}$. 
eine sich personell, organisatorisch und in ihrer Funktionswahrnehmung wandelnde Volkskammer.

Somit bestätigt der Fall der Volkskammer auch Oberreuters Position: Die unter dem Primat des Marxismus-Leninismus bestellte Volksvertretung und ihre Abgeordneten konnten nicht die Repräsentanten der sich durch die Proteste des Herbst 1989 konstituierenden liberalen Zivilgesellschaft sein, sondern eher der am 7. Dezember 1989 erstmals zusammentretende Zentrale Runde Tisch. So kann es nicht verwundern, dass die Volkskammer innerhalb weniger Wochen in die Defensive geriet und sich nach nur kurzer Gegenwehr weitgehend in die ihr bis zu ihrer ersten freien Wahl zugeschriebene Aufgabe fügte: die formaljuristische Umsetzung der am Runden Tisch und in der „Regierung der nationalen Verantwortung“ gefassten Beschlüsse, um die Voraussetzung für die erste freie Volkskammerwahl zu schaffen ${ }^{129}$ - und damit auch für die tatsächliche Verwirklichung des Parlamentarismus in der Deutschen Demokratischen Republik.

129 Vgl. Klemens Semtner, a.a.O. (Fn. 66), S. 99 - 106; Uwe Thaysen, a.a.O. (Fn. 66), Wer war das Volk, S. 277 - 282; ders., a.a.O. (Fn. 66), Wo blieb das Volk, S. 109 - 116.

\title{
Das ungeschriebene amtliche Handbuch der 10. Volkskammer der DDR 1990
}

\author{
Gunnar Peters
}

Parlamente präsentieren sich der Öffentlichkeit in Form von Handbüchern, die insbesondere persönliche Angaben zu allen Abgeordneten enthalten. Die am 18. März 1990 demokratisch gewählte 10. Volkskammer der DDR löste sich am 2. Oktober 1990 auf, ohne dass ein amtliches Handbuch erschien. Erst zehn Jahre später lag ein von Christopher Hausmann erarbeitetes „Biographisches Handbuch“ dieser Volkskammer vor. Nach seinen Angaben sind die Pläne für ein Handbuch seinerzeit „durch die tagespolitischen Ereignisse und die sich abzeichnende Wiedervereinigung obsolet"1 geworden. In einem Beitrag für diese Zeitschrift machte Hausmann außerdem Kostengründe geltend. ${ }^{2}$ Ein Blick in das überlieferte Schriftgut offenbart ein vielschichtigeres Bild: Vor allem die Abgeordneten selbst haben das Erscheinen des Handbuches verhindert.

Von der zweiten bis zur neunten Wahlperiode war für die sozialistische Volkskammer ein amtliches Handbuch veröffentlicht worden, zuletzt 1987. ${ }^{3}$ Noch vor dem 18. März 1990

1 Christopher Hausmann, Biographisches Handbuch der 10. Volkskammer der DDR (1990), Köln u.a. 2000, S. IV.

2 Christopher Hausmann, Die 10. Volkskammer der DDR 1990: Elitenpool der ersten Stunde, in: ZParl, 31. Jg. (2000), H. 3, S. 527 - 541, S. 527.

3 Vgl. Sekretariat der Volkskammer (Hrsg.), Die Volkskammer der Deutschen Demokratischen Republik: 9. Wahlperiode, Berlin (Ost) 1987. 